\title{
Fabry-Pérot Fiber-Optic Sensors for Physical Parameters Measurement in Challenging Conditions
}

\author{
Éric Pinet \\ $R \& D$ Department, FISO Technologies inc., 500-195 Avenue Saint-Jean-Baptiste, QC, Canada G2E 5R9 \\ Correspondence should be addressed to Éric Pinet, eric.pinet@fiso.com
}

Received 30 January 2009; Accepted 2 June 2009

Recommended by Christos Riziotis

Optical fiber sensors have unique advantages and distinctive features that make them very attractive for many applications especially those involving challenging conditions where other traditional electrical sensors usually fail. Among the commercially available optical fiber sensors, the Fabry-Pérot sensing technology is probably the most versatile and the most interesting one since a relatively low-cost universal signal conditioner could easily read compatible Fabry Pérot sensors measuring different physical parameters such as strain, temperature, pressure, displacement, or refractive index. This papers details the numerous advantages of this optical sensing technology and also summarizes the operating modes of commercially available signal conditioners and sensors.

Copyright (C) 2009 Éric Pinet. This is an open access article distributed under the Creative Commons Attribution License, which permits unrestricted use, distribution, and reproduction in any medium, provided the original work is properly cited.

\section{Introduction}

When someone hears the terms "optical fiber," usually the first association that comes to mind is often related to a fast and high-volume modern communication link that helps the expansion of the exploding information data demand created by our "image society driven", by the democratization of the Internet and other new communication technologies. Some others will also associate the optical fibers to their glowing plastic Christmas tree, illuminated by a constellation of stars created at the tip of a myriad of plastic optical fibers which are transmitting the light of a changing color lamp.

Actually very few will think that optical fibers could also be at the core of a sensor technology that already started to take place in our day-to-day life. In that case the fiber optic remains a physical link for the light that travels between the sensor and the signal conditioner, but how the information is coded and translated into useful data will depend on the used technology.

In the last few decades tremendous work has been done by academics and the industry to develop sensing technologies using light properties propagating in optical fibers [1-3] allowing measurements particularly in challenging conditions.
The candidate technologies for monitoring a physical parameter with optical fiber sensor (OFS) can be classified in four main categories relying on the position where the parameter is measured: single-point, long gage, quasidistributed and distributed [4]. In single-point sensing, the measurement is performed at a defined point along the fiber, usually at or near its end, as the case for most FabryPérot (F-P) sensors. In long gage sensor the measurement is performed along usually $10 \mathrm{~cm}$ to $10 \mathrm{~m}$ sections of optical fiber, such as for the SOFO system [5]. For quasi-distributed OFS, a limited number of sensing points are physically located on the same fiber, as the case for fiber Bragg grating (FBG) dominating this type of sensor. In fully distributed OFS, the optical fiber itself acts as a sensing medium, which could be used to discriminate different positions of the measured parameter along the fiber such as for Raman or Brillouin distributed sensors.

Among the various technologies now commercially available, the Fabry-Pérot white-light interferometry is probably one of the most interesting for point-sensing applications since it is a low-cost solution for multiparameters measurement. This paper describes the advantages of this type of sensor and interrogation technology that is suitable for physical parameters measurement in challenging conditions. 


\section{Why Use Fabry-Pérot Fiber-Optic Sensors?}

Measuring a physical parameter such as temperature, pressure, or strain could sometimes become a real challenge if the environment is not suitable for traditional sensors such as electrical sensors. For instance, in the presence of strong electromagnetic (EM) or microwave (MW) interferences, it could be a headache to shield a thermocouple for accurate and reliable temperature measurement. With an optical sensor such trouble is simply avoided since the encoded light confined in the optical fiber core is not affected by any electromagnetic interference (EMI). So any time such interferences are present in a given application, the optical sensing technology is probably the best solution to select, if not sometimes the only one. For instance, without an optical fiber sensor it would be very tricky to monitor your hotdog heating in your microwave oven, and you will probably never know that your sausage is hot burning while your bun is still frozen-until you take a bite. If you were a food packaging engineer trying to optimize the heat transfer of a complex microwavable TV dinner or if you were a designer or a programmer of MW oven, having real time temperature data during the MW process may be much more that a simple gadget for hot-dog fans. You may thus probably appreciate using an instrumented microwave work station (MWS) equipped with OFS. As an example, Figure 1 shows temperature experimental data obtained for the heating of a $1 \mathrm{~L}$ water bath in a $1200 \mathrm{~W}$ commercial microwave oven for the calculation of its peak power according to the protocol described in international standard document [6]. The temperature was measured from 3 different FOT$\mathrm{L}$ temperature sensors connected to OSR interrogator which allows to calculate MW power, even during MW heating. The peak MW power measured experimentally confirms the $1200 \mathrm{~W}$ value of the oven manufacturer.

Same measurement problems occur if you were a chemist trying to control critical parameters such as pressure or temperature during a microwave chemistry process, an engineer supervising radio-frequency (RF) wood drying, a physician monitoring the temperature of a critical care patient undergoing a magnetic resonance imaging (MRI) scan, or even if you were a surgeon burning selected tissues with RF instrumented minimally invasive catheters: without an optical sensor you may face big difficulties. Even in extreme environments such as those encountered into nuclear reactors where strong radiations are present, the optical sensors could provide an attractive alternative solution $[7,8]$.

EMI may not always be constantly present during the measurement to justify the use of OFS. If you were a civil engineer selecting a solution for monitoring a bridge, a dam, or other types of structures over several decades, would you take the risk that a lightning strike destroys or damages permanently your electrical sensors network buried into the structure, or would you use an optical technology [9-14] proven to be unaffected by such unpredictable events? Also as a critical care patient, you would probably feel more secure if you knew that your vital signs such as pressure and temperature are measured with optical sensors [4] that will

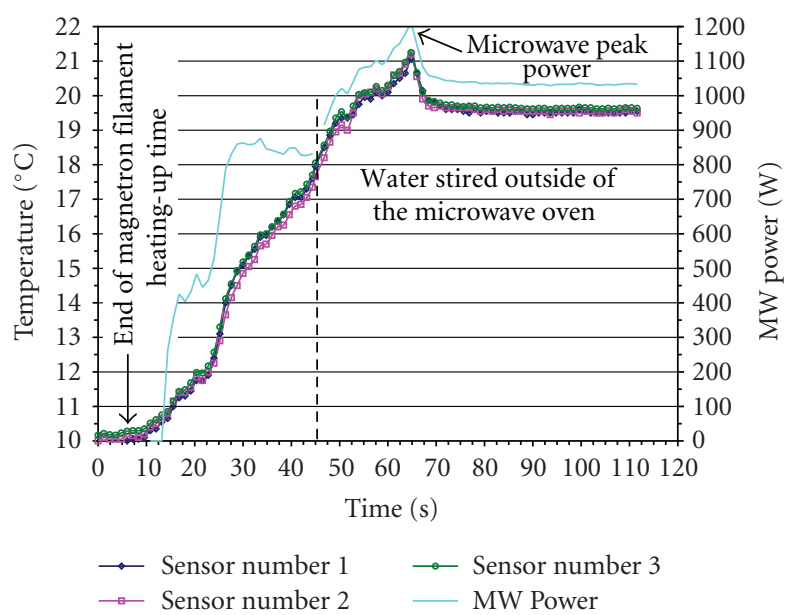

Figure 1: In situ measurement of $1 \mathrm{~L}$ water bath temperature inside a $1200 \mathrm{~W}$ microwave with 3 FOT-L temperature sensors and OSR interrogator from FISO Technologies allows oven power calculation even during microwave heating. The microwave peak power $(1200 \mathrm{~W})$ is determined using the protocol described in the IEC-60705 international standard. The end of magnetron filament heating-up time is detected by the start of water bath temperature increase. After 45 seconds (vertical dashed line) when the heating program stops, the door is open and the water is stirred for better temperature uniformity. The temperature rises to a maximum that is used to define the microwave power as stated in the IEC-60705 international standard.

not give false readings even in the presence of possible EMI generated in modern hospitals crowded by various electrical equipments.

The insensitivity to EMI is not the only advantage of optical sensors. Inversely, since no electricity is used to interrogate the sensor only powered by light, such device is intrinsically safe and naturally totally explosion proof. This may be a great feature if you are working with inflammable gases or liquids or if you want to avoid that the sensor could be a source of electrical discharges or EM field perturbation. For instance, liquid natural gas (LNG) reservoirs are obviously excellent candidates for fiber optical strain, pressure, or temperature sensors. Many other applications could be found in the chemical processing industry or in military applications such as the one involving explosive materials or missiles control.

Another corollary of the fact that light instead of electricity carries the useful information between the sensor and the signal conditioner is the fact that very low attenuation occurs along the optical fiber. Indeed this advantage is truly unique if measurements have to be done in remote locations, as it is often the case for large civil structures such as pipe-lines [15], bridges, tunnels, or dams. Furthermore if the optical fiber has to cross zones with strong EMI, no problem will occur in the transmitted measurement, which is not the case with electrical transmission. For instance, with the Fabry-Perrot sensing interferometry developed by FISO Technologies, up to $5 \mathrm{~km}$ (expandable to $12 \mathrm{~km}$ with custom long-range signal conditioner) could separate measuring 


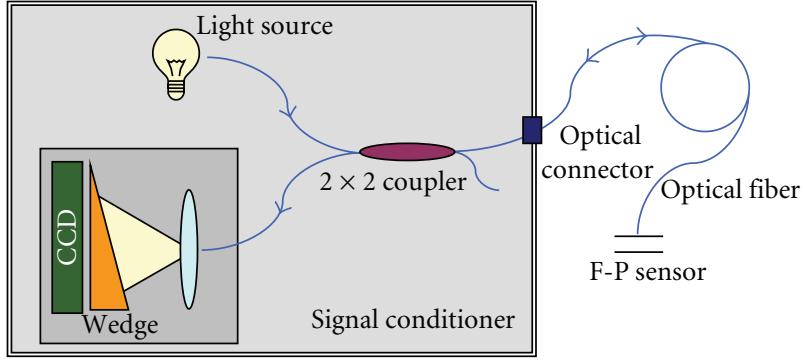

Figure 2: Schematic description of the F-P absolute measurement signal conditioner using white-light interferometry.

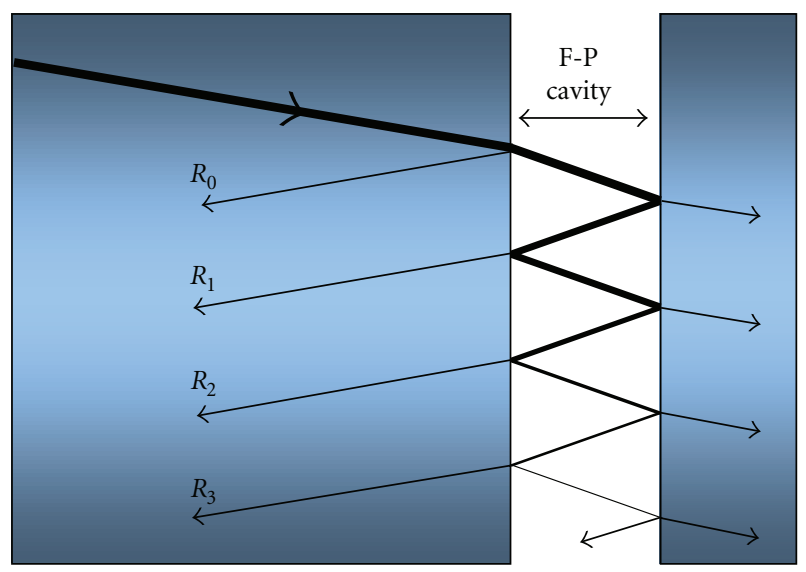

Figure 3: Schematic representation of F-P sensing interferometer showing ray traces obtained from a selected incident angle light beam propagating in the optical fiber core.

and recording locations with the same outstanding sensor sensitivity and accuracy.

Compared to copper wires, glass optical fibers are lighter, and such a property may become an interesting feature for applications where lightweight sensor solutions are a concern such as in aeronautics and more crucially in spatial applications. The fact that glass is a stable inert material that does not corrode is also an advantage for long-term applications in outdoor conditions such as typical for most of civil engineering applications.

Contrary to electrical sensors, most of the optical sensors do not have metal in their construction. In some cases, metals could be used for packaging when this does not present a problem, but an all-glass and polymer sensor has great advantages when interferences with an electromagnetic field are not desirable such as in hospitals during MRI investigations. The materials used in the sensor construction and packaging are also generally biocompatible and chemically inert, which makes optical sensors perfectly suitable for medical or harsh chemical environments applications. Besides its chemical resistance, glass is also very resistant to temperature providing an additional advantage that is very attractive to a lot of challenging applications. Actually, OFS compatible with temperature ranging from cryogenic up to about $450^{\circ} \mathrm{C}$ is commercially available.
TABLE 1: Summary of F-P OFS technology advantages.

\begin{tabular}{lcc}
\hline Design & Environment & Other \\
\hline Inert materials & Cryogenic to high & Biocompatibility and \\
(glass...) & temperature & sterilization \\
Robust packaging & Chemical resistance & Symmetry axis \\
Light powered & EMI insensitive & Intrinsically safe \\
Small size & In situ monitoring & Light weight \\
Low attenuation & Remote location & Low -cost OFS \\
\hline
\end{tabular}

If temperature is combined with harsh chemical environment such as the case in oil and gas wells or with strong EMI such as encountered in plasma research, the optical fiber sensing solution is even more attractive.

Besides all the named advantages of F-P OFS which are summarized in Table 1, the one that is probably among the most interesting is the ultraminiature size that can be achieved for such sensors without sacrificing the sensor reliability and precision. Combined with its small size, the geometry of the sensor, which is usually axial, is sometimes an additional interesting advantage. For instance, it is possible to monitor strain in a small bolt almost without changing its mechanical properties: a small hole drilled in its center and filled with a $\varnothing 230 \mu \mathrm{m}$ OFS glued strain sensor (FOS-N) will certainly give more accurate measurements than an electrical flat strain gage that could only be installed by creating a flat surface on the bolt and that will definitely create a weak point thus biasing the true strain measurement.

The sensor miniaturization accessible with OFS allows accurate in situ measurements exactly where the physical parameter has to be detected in sometimes hard-to-reach areas. This has a major impact in a lot of industries, especially in the medical applications involving minimally invasive diagnostics and therapies $[16,17]$. Pressure, temperature, and strain OFS manufactured have found many market niches where their size and reliability were key requirements. For instance, the FOP-MIV pressure sensor (see Figure 4), which is currently mass-produced and assembled using a fully automated assembly line with top quality control, meets all requirements necessary for integration into medical devices [18]. It is thus suitable for many medical applications where miniaturization and sensor global reliability are essential. For instance, it can be integrated into a catheter used for intra-aortic balloon pumping (IABP) therapy [19] which is a life-supporting therapy used in many heart surgical situations [20-22]. The miniature optical sensor can supply an accurate pump triggering signal directly from the in situ measured aortic pressure waveform (such as the one simulated in Figure 5(a)). The IABP therapy integrating optical pressure sensors is simplified and is also more reliable since the diameter of the IABP catheters is now reduced to sizes not available with conventional IABP catheters using fluidic transduction pressure monitoring. Such highly valuable miniaturization reduces significantly the occurrence of ischemia, which is the highest risk of this therapy [23]. 


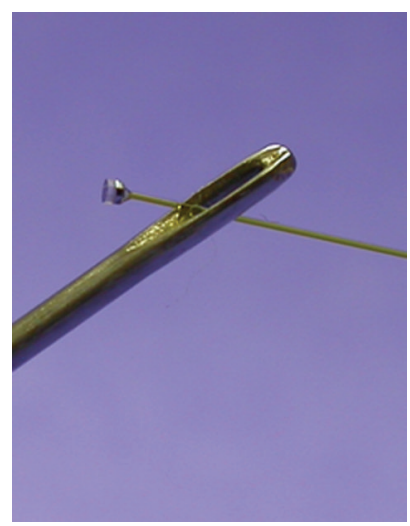

(a)

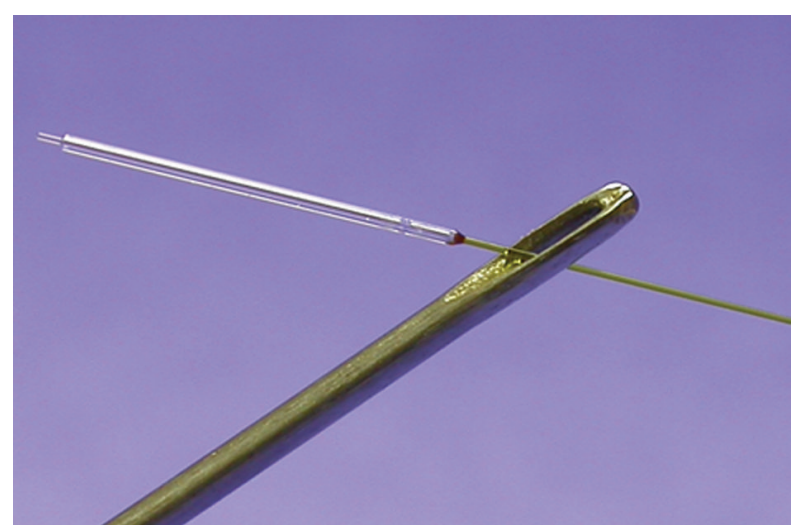

(b)

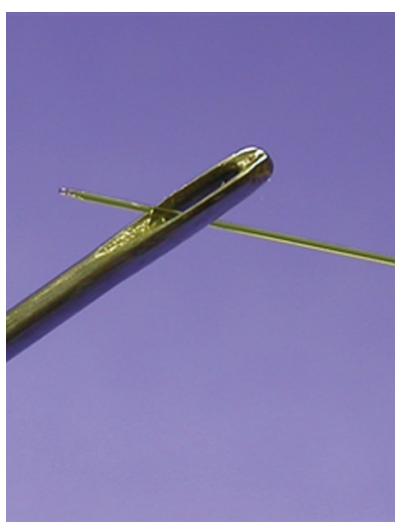

(c)

FIGURE 4: Examples of bare fiber-optic sensors for medical applications commercially available from FISO Technologies. (a) pressure sensor FOP-MIV suitable for mass production $(\varnothing 550 \mu \mathrm{m})$, (b) temperature sensor FOT-L $(\varnothing 800 \mu \mathrm{m})$, (c) temperature sensor FOT-HERO $(\varnothing$ $210 \mu \mathrm{m})$.

Additional miniaturization of pressure sensor with diameter similar to the one of an optical fiber $(\varnothing 125 \mu \mathrm{m})[24,25]$, currently under development, will push further away the limits achievable with OFS and will open new avenues for minimally invasive in situ measurements.

In some cases the selection of an OFS could be driven only by one unique advantage provided by the optical technology, but in some cases a combination of criteria will practically make the choice of OFS the only applicable solution. For instance, in engine testing industry where the motor generates not only a lot of heat in a harsh explosive chemical environment but also a burst of EMI that fools traditional electrical sensors, the use of OFS for monitoring pressure $[26,27]$, strain cycles, or temperature, with minimal perturbation of the system, is probably a wise approach.

\section{How does the Signal Conditioner Work?}

Among the fiber-optic point-sensing commercially available technologies, the Fabry-Pérot white-light interferometry is probably the most attractive one. Its greatest advantage is undoubtedly the fact that it offers a low-cost solution for detection of a large variety of physical parameters such as temperature, pressure, strain/force, displacement, and refractive index. All those parameters could be accurately measured with the same universal signal conditioner that has evolved to meet all industrial robustness criteria.

FISO Technologies actually offers two patented technologies [28-30] that are suitable for interrogating Fabry-Pérot sensors. One provides an accurate measurement of the sensor reading (absolute measurement) while the other gives only a measurement of its variations (relative measurement).

Compared to other fiber-optic sensing technologies requiring costly optical source or spectral analysis equipment such as for interrogating FBG sensors, the Fabry-Pérot technology requires inexpensive and simple system instruments as it will be described in the following sections.
3.1. The Absolute Measurement Sensing Technology. The greatest advantage of the first technology is that the measurement is absolute which means that the sensor could be disconnected and reconnected and still give the same reading without any adjustment. Due to light source and detector limitations, such technology is now limited for sampling rates in the $\mathrm{kHz}$ range which is however suitable for most industrial practical applications (the recently released FPIHS allows a $15 \mathrm{kHz}$ sampling rate).

The principle behind this sensing technology, schematized in Figure 2, is actually quite simple although some technical details have to be considered in order to manufacture such device.

A light source, namely, a bright incoherent light source, is first injected and guided into a multimode optical fiber and then into an input of a $2 \times 2$ coupler which acts as a $50 / 50$ power splitter. One output is linked to the OFS through an optical connector at the signal conditioner front panel. The second output of the coupler is not used or in some configurations could be connected to a light detector for light source monitoring purposes. Then, the light travels through the lead optical fiber until it reaches its tip, where the OFS is assembled.

The core of the OFS is a Fabry-Pérot interferometer that has been actually well known in the optical scientific community for now more than a century [31]. It has been used in many research applications such as physics and astrophysics notably. It is indeed constituted by two parallel, perfectly flat semireflecting mirrors separated by a given gap. The light passing through a first mirror is reflected back and forth a very large number of times between the two parallel planes as shown in Figure 3. However, at each reflection, a small fraction of the incident beam escapes the interferometer creating a large number of parallel beams of light $\left(R_{0}, R_{1}, \ldots, R_{n}\right)$ emerging at the same angle at which they entered the interferometer. In the free space, they could be focused to form an image by a converging lens creating a constructive interference by the multiple beams which produce very bright and sharp interference fringes. Their 


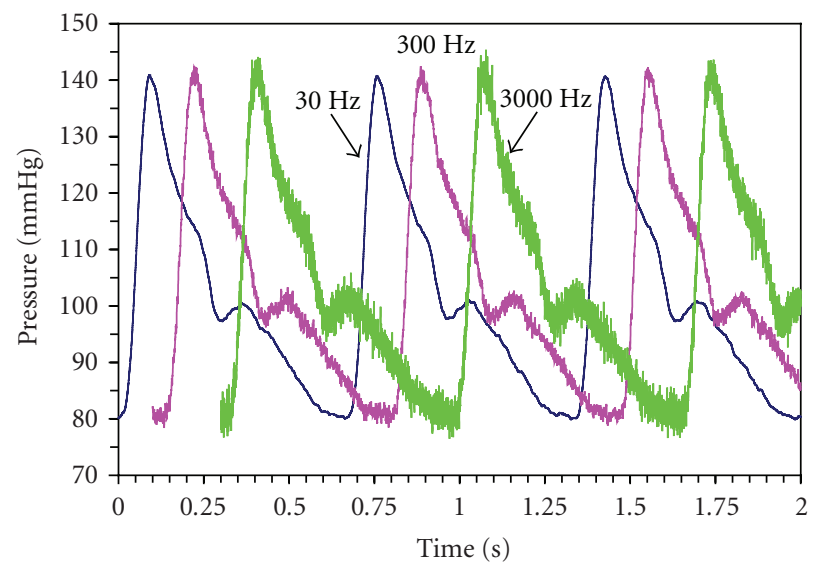

(a)

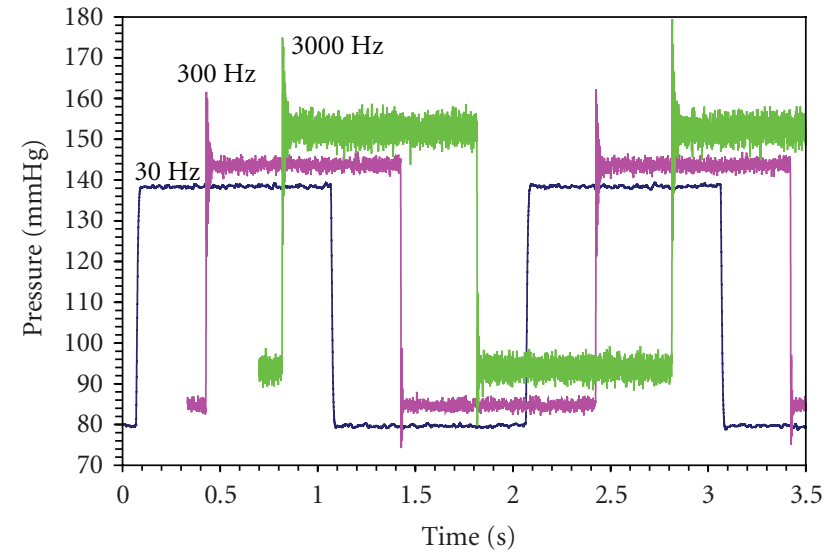

(b)

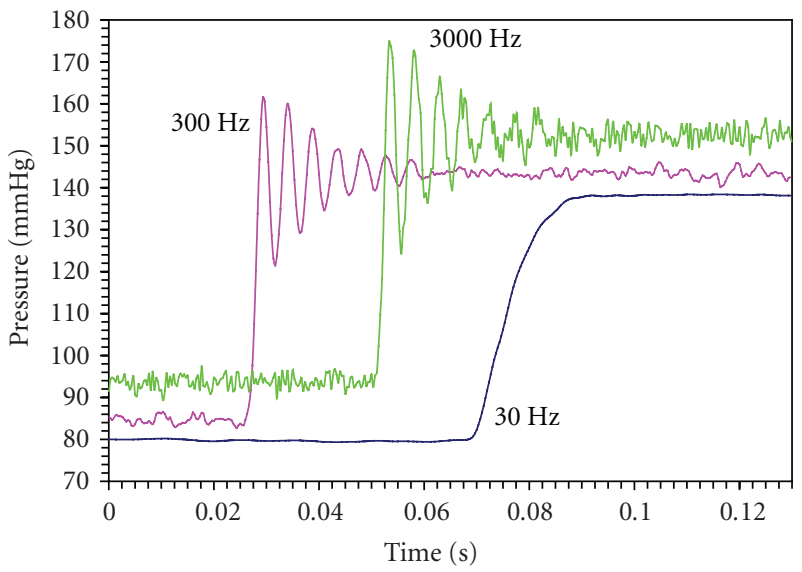

(c)

FIGURE 5: $80-140 \mathrm{mmHg}$ pressure waveforms generated in water with a Bio-Tek 601A pressure simulator and measured by an FOP-M260 pressure sensor connected to a $15 \mathrm{kHz}$ FPI-HS interrogator from FISO Technologies. Acquisition rate is set to $3 \mathrm{kHz}$, and lowpass filter is set to $30 \mathrm{~Hz}, 300 \mathrm{~Hz}$, and $3000 \mathrm{~Hz}$. (a) Normal aorta waveform, $90 \mathrm{bpm}$. (b) $0.5 \mathrm{~Hz}$ square waveform. Graphs have an offset for better clarity. (c) Detail of previous graph with graphs offset for better clarity to show an increasing pressure impulse.

spacing will depend on the optical path (that is related to the distance separating the parallel planes and the refractive index between those planes) and naturally on the light wavelength. Thus if a physical parameter to be measured by the sensor changes the optical path difference (OPD) of the $\mathrm{F}-\mathrm{P}$ interferometer, the light escaping the F-P interferometer will be encoded according to this variation.

In the fiber-optic version, the light emerging from the F-P interferometer is not directly focused on a plane to give the interferences mentioned above, but the light beams are rather reinjected into the optical fiber from which they originally came from and they travel back, entering the signal conditioner at the optical connector level. Then, the light is again separated by the $2 \times 2$ coupler into two fibers. The light directed back to the light source is lost whereas the other fiber directs the light toward an optical box where the light is spread over a Fizeau wedge that reconstructs the interference pattern which is physically recorded using a charge coupled device (CCD). Due to the fact that whitelight is used, all wavelengths are present, and thus destructive interferences occur except for the zero order where all wavelengths are actually constructive. Thanks to the wedge that creates a linear variation of thicknesses, the crosscorrelated interference pattern has a maximum intensity at the exact position the optical path difference equals the one created at the F-P sensor, and few lower intensity peaks symmetrically disposed around the central peak (as given by the interferometer cross-correlation function). Thus finding the sensor OPD related to the physical parameter to be measured simply consists of finding the position of the maximum peak in the CCD interference pattern. This robust interferometric method allows accurate and precise F-P cavity length measurement with sub-nanometer range precision over several decades of micrometer span, thus giving a very interesting dynamical range.

3.2. The Relative Measurement Sensing Technology. The second technology, commercialized under the Veloce brand name, only provides a relative measurement: the sensor has to be referenced each time it is optically connected. This technology is best fitted for monitoring very fast occurring 


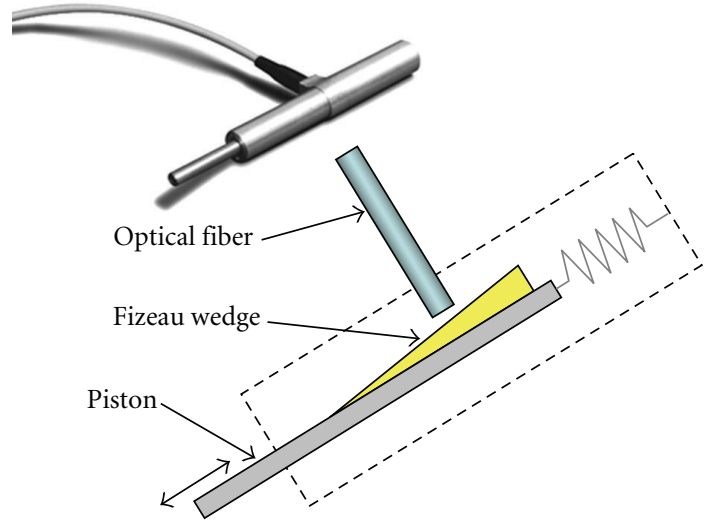

FIgUre 6: Photo (top left) and schematic representation of FOD displacement sensor from Fiso Technologies. As the piston is moving, the optical fiber faces different thicknesses of the Fizeau wedge.

events such as explosions or blasts [32], for instance, or phenomena requiring very high resolution. Thanks to the use of more efficient light sources and detectors, the acquisition rate is much faster than with the other technology: typically $0.2 \mathrm{MHz}$ but it could be extended to $2 \mathrm{MHz}$ if data postprocessing is possible.

In this technology, the light source is actually a bright light emitting diode (LED) that has a narrow wavelength band. Similarly to the previous method, the light is injected into an optical fiber and is guided to the F-P sensor via a $2 \times 2$ coupler and an optical connector at the signal conditioner front panel. Once returning from the sensor, the coded-light passes again through the connector and the $2 \times 2$ coupler, but instead of being directed to an optical box like previously, it is rather again separated into two optical fibers by another $2 \times 2$ coupler. Each fiber is directed to a calibrated F-P cavity mounted in front of a photodetector. The calibrated F-P cavities are actually selected so that the two detectors record signals in phase quadrature: that is, if one photodetector records a cosine signal, the other would record a sine signal for a linear variation of the physical parameter that is measured.

After referencing the sensor at the beginning of the measurement which means selecting an arbitrary phase in the periodic transfer function, the signal treatment algorithm allows tracking the sensor position within this periodic function. Therefore the sensor variation is not limited to a single period, and several orders could be passed without any problem. The only limitation is that the sensor order change should not be faster than the acquisition rate so that the tracking can be performed continuously. In most experimental cases this is obviously not a problem.

\section{How Does the Fabry-Pérot Sensor Work?}

Most of the Fabry-Pérot optical sensors are constructed around the same idea: they have an F-P interferometer whose OPD changes according to the physical parameter they are designed to measure. The sensor OPD is accurately measured by the signal conditioner of both described technologies absolute or relative measurement. Thanks to an appropriate sensor calibration, this OPD is converted into the appropriate unit corresponding to the sensor type to display a comprehensive value to the end-user.

Although several differences do exist between various commercially available sensor models, they could be briefly summarized by physical parameters categories.

4.1. Strain Sensors. The core sensing part of strain sensors is constituted by two glass fibers, which are ended by perfectly flat surfaces facing each other to constitute the F-P cavity with a given length. Thanks to a microcapillary glass tube that permanently fixes each fiber at a well-defined point few millimeters away from the F-P cavity, this alignment is constantly maintained. The ratio of the F-P cavity length variation to the distance separating the two fixing points is simply the strain measured by the sensor (with unit $\mu \varepsilon$ ).

To measure the strain of a material, the sensing capillary tube has to be glued or embedded onto or within the material to be studied. Once the material is deformed by a load or by a thermal dilatation, this deformation is transmitted to the capillary tube and thus to the F-P cavity whose length is changing accordingly and is precisely measured by the signal conditioner.

Due to the geometry of this sensor, it is practically not sensitive to transversal strain: only axial strains are thus detected. This is an additional great advantage over traditional electrical strain gages.

With its design, since the glass material used for the optical fibers forming the F-P cavity and the capillary tube is similar, such strain sensor is almost not affected by thermal dilatation: thermal expansion of the fibers is always compensated by thermal expansion of the capillary tube. The only place where such compensation does not occur is at the F-P level, which means that only few micrometers are not thermally compensated. Compared to other commercial OFS strain sensors such as fiber Bragg grating (FBG) that are also commonly used in the industry, Fabry-Pérot strain sensors are at least an order of magnitude less thermally sensitive, making it possible for some applications to avoid temperature measurement for thermal correction purposes.

Beside the bare sensor configuration that has been described here above, FISO Technologies also manufacture configurations where the strain sensor has been integrated into robust packaging that could be either welded (SFO$\mathrm{W}$ sensor) on steel structure or directly embedded into cast concrete or similar materials (EFO sensor). Several dynamical ranges are available as standard products $( \pm 1000 \mu \varepsilon$, $\pm 2500 \mu \varepsilon$, and $\pm 5000 \mu \varepsilon$ ), but customization of this sensor could easily be done to take the best advantage of the whole dynamical range of the signal conditioners. For all these sensors the typical resolution (which also depends on the interrogator used) is $0.01 \%$ of full scale.

4.2. Temperature Sensors. Two types of F-P temperature sensors are available commercially: capillary type and refractive index type (see Figure 4). 
The design of the capillary type temperature sensor is actually very similar to the above described strain sensor: two flat-ended fibers are assembled in a glass capillary tube to form an F-P cavity. But contrary to the strain sensor, the material of one fiber is selected to have a high coefficient of thermal expansion (CTE). The fiber thermal variation is thus not anymore compensated by the one of the capillary tube.

Also, this tube is encapsulated into a bigger capillary tube to prevent the fact that the sensor sensing part could be affected by strains transmitted through the packaging.

When temperature increases, the thermally sensitive fiber expands, reducing the F-P cavity length. Thanks to factory calibration, this length variation is translated into temperature value.

Several packaging and thermal ranges are available for FOT-L sensors and could be selected depending on the specific needs of the application. Response time of the sensor will of course depend on the selected packaging, but less than 0.5 second is a typical value for a packaged sensor and about 1 millisecond for a bare sensor. A typical accuracy for this sensor is $0.3^{\circ} \mathrm{C}$ for a medical temperature range $\left(20^{\circ} \mathrm{C}\right.$ to $\left.85^{\circ} \mathrm{C}\right)$ and $1^{\circ} \mathrm{C}$ for an industrial temperature range $\left(-40^{\circ} \mathrm{C}\right.$ to $\left.300^{\circ} \mathrm{C}\right)$.

Another type of temperature sensor is also available. This time, instead of material thermal dilatation, temperature dependent refractive index is rather used to change the OPD of the F-P sensor. A tiny chip of a semiconductor material with high thermal refractive index dependence and two semireflective surfaces constituting an F-P cavity is assembled at the tip of the lead optical fiber. This solid compact design is actually the smallest optical fiber temperature sensor available on the market ( $150 \mu \mathrm{m}$ square). Its sensitivity is about one order of magnitude lower than the capillary type temperature sensor, but due to its extremely low thermal mass, its response time is better than $5 \mu$ s for a bare sensor which makes this sensor extremely interesting for fast temperature changes monitoring or for precise spatial point temperature mapping applications.

4.3. Pressure Sensors. Fabry-Pérot pressure sensors, also known as piezometers especially in the civil engineering industry, usually have a similar design. A reflecting deformable membrane is assembled over a generally vacuumed cavity made in a transparent material, thus forming so to speak a small drum. The bottom of the cavity and the inner flat surface of the flexible membrane are forming the sensing F-P cavity. When pressure is applied, the membrane is deflecting toward the bottom of the cavity thus reducing the F-P cavity length. With appropriate sensor calibration, each cavity length corresponds to a pressure value that is displayed with selected appropriate unit.

Several ranges (from $\pm 40 \mathrm{kPa}$ to $0-70 \mathrm{MPa}$ relative to atmospheric pressure) are also available with various packaging alternatives designed to fit various applications. Two major pressure sensor families could be selected among available products at FISO Technologies: the FOP-C is a bulky robust sensor with a flexible metal membrane mainly designed for industrial applications requiring medium to high-pressure ranges and the FOP-M family which offers miniature sensors $(\varnothing \sim 0.25-0.5 \mathrm{~mm})$ manufactured using well-established and well-controlled photolithographic techniques. In this case, the flexible membrane is made of a thin silicon layer bonded to a glass with the cavity. It fits most applications where size and accuracy are important issues. It is available from $40 \mathrm{kPa}$ to $20.7 \mathrm{MPa}$ (full range) and has the highest sensitivity. Some other designs are also available where the optical fiber axis and the flexible membrane are in parallel planes (FOP-MS and FOP-MSL). Such configurations are sometimes useful when routing the optical fiber is difficult in a given application.

Figure 5 shows the waveforms obtained from a $260 \mu \mathrm{m}$ diameter FOP-M260 pressure sensor measured by an FPIHS interrogator. This interrogator can have a software configurable acquisition rate up to $15 \mathrm{kHz}$. In the Figure 5 an acquisition rate of $3 \mathrm{kHz}$ was selected, and different programmable lowpass filters were applied to adjust the output bandwidth. On Figure 5(a), showing a simulated typical $80-140 \mathrm{mmHg}$ aortic pressure waveform [19], it could be seen that a $30 \mathrm{~Hz}$ filter provides a smoother profile without distorting the experimental data. If such bandwidth is sufficient for human blood pressure monitoring, it may not be the case for small animals which have faster heartbeat [16]. When higher frequencies are present such as in the $0.5 \mathrm{~Hz}$ square waveform presented in Figure 5(b), applying a higher filter allows visualization of fine signal structures that otherwise are smoothed out. It could be seen on the zoom showing the abrupt pressure increase in Figure 5(c) that a $\sim 200 \mathrm{~Hz}$ bouncing damping fluctuation is generated in the system by the Bio-Tek 601A pressure simulator at each drastic pressure change. A $300 \mathrm{~Hz}$ filter is in that case more appropriate than a $30 \mathrm{~Hz}$ filter that eliminates fast pressure variations and is better than a $3 \mathrm{kHz}$ filter (corresponding to no filter since acquisition rate was set to $3 \mathrm{kHz}$ ) since data is smoother due to filter averaging. Adapting acquisition rate and output bandwidth (such as possible with the FPI-HS interrogator) allows therefore optimal signal processing.

4.4. Refractive Index Sensors. In some respects, refractive index sensors have a design that is derived from pressure sensors. But in that case the top membrane is not flexible, and the cavity is opened on two sides instead of being sealed and vacuumed. The physical dimension separating the two flat planes, creating the F-P cavity is maintained fixed and opened to the external medium, and so any fluid (gas or liquid) that enters the cavity could change its refractive index and thus will change the OPD of the F-P cavity that is accurately measured by the signal conditioner. Refractive indeces from 1.000 to 1.700 , covering a wide range suitable for most liquids and gasses, are typically accessible with a standard FRI sensor with a typical resolution of $10^{-4}$ RIU. However, custom design could allow higher refractive index ranges if necessary.

Since the open F-P cavity is only few micrometers wide, very opaque fluids could be measured. For instance, you could easily see the increase of refractive index of your black coffee with milk as you dissolve sugar in it. The only 
limitation is that the fluid should easily penetrate the F-P cavity, and so viscous fluids or fluids with bubbles will thus be more difficult to measure. But, contrary to other refractive index techniques based on diffraction angles measurements, the F-P refractive index sensor is not much affected by surfaces contamination since it actually measures truly bulk refractive index back and forth through the F-P cavity; so effects of surface contaminant layers are usually negligible compared to the whole optical path.

4.5. Displacement Sensors. F-P displacement sensors have a design quite different from all other described sensors. A Fizeau wedge mounted on a spring loaded axis is positioned in front of the extremity of the lead optical fiber as shown in Figure 6. As the axis is moving, the fiber extremity faces the wedge with variable thickness F-P cavity that could be related, with appropriate calibration, to the linear axis position.

On the standard FOD displacement sensor (see Figure 6), with a range of $20 \mathrm{~mm}$ and an accuracy of $2 \mu \mathrm{m}$, the optical fiber is normal to the displacement axis. Longer ranges displacement sensors and special sensors with optical fiber in the same direction than the displacement axis are also available.

\section{Conclusion}

As discussed in this paper, Fabry-Pérot optical sensing technology offers a real variety of sensors for physical parameters (such as strain, temperature, pressure, refractive index, displacement, etc.) that could be interrogated with two commercial families of universal signal conditioners, giving either fast (up to the $\mathrm{kHz}$ range) absolute measurements or ultrafast (up to the $\mathrm{MHz}$ range) relative measurements.

Such optical sensors have unique advantages that make them suitable for many challenging conditions that sometimes are impossible to address with conventional electrical sensors. They are particularly well adapted in various environments, for instance, in the presence of strong EMI, when there is a risk of explosion or of inflammability, when the temperature is too low or too high or when the chemical environment is really harsh. When other sensors are too bulky or too heavy, when hard-to-reach measuring spots are involved, or when measuring equipments have to be in remote locations, the Fabry-Pérot optical fiber sensors should be considered as a practical solution to problems which sometimes are puzzling the engineers.

One additional reason worth considering is that this field-proven optical sensing technology, commercialized for now more than a decade, is one of the lowest costs on the market. Actually with the recent introduction of its new Evolution Platform accepting OEM-ready snap-on DINrail modules, FISO Technologies now offer to the engineers and scientific community the cost-reduction and quality benefits from high-volume OEM modules and sensors, without sacrificing the possibility for customization and expandability.
With this new democratization of the access to this original and unsurpassed sensing technology for challenging conditions, one can bet that new opportunities and innovative applications will flourish in the very near future, making those optical sensors more and more present in our day-today life.

\section{References}

[1] D. A. Krohn, Fiber Optic Sensors: Fundamentals and Applications, Instrument Society of America, Research Triangle Park, NC, USA, 2nd edition, 1992.

[2] W. J. Bock, I. Gannot, and S. Tanev, Eds., Optical Waveguide Sensing and Imaging, NATO Science for Peace and Security Series B: Physics and Biophysics, Springer, Berlin, Germany, 2006.

[3] S. Yin, P. B. Ruffin, and F. T. S. Yu, Eds., Fiber Optic Sensors, CRC Press, Boca Raton, Fla, USA, 2nd edition, 2008.

[4] É. Pinet, C. Hamel, B. Glišić, D. Inaudi, and N. Miron, "Health monitoring with optical fiber sensors: from human body to civil structures," in Health Monitoring of Structural and Biological Systems, vol. 6532 of Proceedings of SPIE, San Diego, Calif, USA, 2007.

[5] D. Inaudi, "Field testing and application of fiber optic displacement sensors in civil structures," in Proceedings of the 12th International Conference on Optical Fiber Sensors (OFS '97), vol. 16 of OSA Technical Digest Series, pp. 596-599, Williamsburg, Va, USA, 1997.

[6] International standard IEC 60705:1999 + A1:2004 + A2:2006, "Household microwave ovens-methods for measuring performance," Edition 3.2 (2006-03).

[7] H. Liu, D. W. Miller, and J. Talnagi, "Gamma radiation resistant Fabry-Pérot fiber optic sensors," Review of Scientific Instruments, vol. 73, no. 8, p. 3112, 2002.

[8] H. Liu, D. W. Miller, and J. W. Talnagi, "Performance evaluation of Fabry-Pérot temperature sensors in nuclear power plant measurements," Nuclear Technology, vol. 143, no. 2, pp. 208-215, 2003.

[9] P. Choquet, R. Leroux, and F. Juneau, "New Fabry-Pérot fiber-optic sensors for structural and geotechnical monitoring applications," Transportation Research Record, no. 1596, pp. 39-44, 1997.

[10] M. Quirion and G. Ballivy, "Concrete strain monitoring with Fabry-Pérot fiber-optic sensor," Journal of Materials in Civil Engineering, vol. 12, no. 3, pp. 254-261, 2000.

[11] P. Choquet, M. Quirion, and F. Juneau, "Advances in FabryPérot fiber optic sensors and instruments for geotechnical monitoring," Geotechnical News, vol. 18, no. 1, pp. 35-40, 2000.

[12] M. Quirion and G. Ballivy, "Laboratory investigation on Fabry-Pérot sensor and conventional extensometers for strain measurement in high performance concrete," Canadian Journal of Civil Engineering, vol. 27, no. 5, pp. 1088-1093, 2000.

[13] B. Benmokrane, M. Quirion, E. El-Salakawy, A. Debaiky, and T. Lackey, "Fabry-Pérot sensors for the monitoring of FRP reinforced bridge decks," in Nondestructive Evaluation and Health Monitoring of Aerospace Materials and Composites III, vol. 5393 of Proceedings of SPIE, pp. 95-102, San Diego, Calif, USA, July 2004.

[14] B. Glišić and D. Inaudi, Fibre Optic Methods for Structural Health Monitoring, John Wiley \& Sons, New York, NY, USA, 2007. 
[15] D. Inaudi and B. Glišić, "Long-range pipeline monitoring by distributed fiber optic sensing," in Proceedings of the 6th International Pipeline Conference (IPC '07), pp. 763-772, Calgary, Canada, September 2007.

[16] C. Hamel and É. Pinet, "Temperature and pressure fiberoptic sensors applied to minimally invasive diagnostics and therapies," in Optical Fibers and Sensors for Medical Diagnostics and Treatment Applications VI, vol. 6083 of Proceedings of SPIE, San Jose, Calif, USA, February 2006.

[17] É. Pinet, "Medical applications: saving lives," Nature Photonics, vol. 2, no. 3, pp. 150-152, 2008.

[18] É. Pinet and C. Hamel, "True challenges of disposable optical fiber sensors for clinical environment," in Third European Workshop on Optical Fibre Sensors, vol. 6619 of Proceedings of SPIE, pp. 1-4, Napoli, Italy, July 2007.

[19] É. Pinet, A. Pham, and S. Rioux, "Miniature fiber optic pressure sensor for medical applications: an opportunity for intra-aortic balloon pumping (IABP) therapy," in 17th International Conference on Optical Fibre Sensors, vol. 5855 of Proceedings of SPIE, pp. 234-237, Bruges, Belgium, August 2005.

[20] G. A. Maccioli, W. J. Lucas, and E. A. Norfleet, "The intraaortic balloon pump: a review," Journal of Cardiothoracic Anesthesia, vol. 2, no. 3, pp. 365-373, 1988.

[21] D. F. Torchiana, G. Hirsch, M. J. Buckley, et al., "Intraaortic balloon pumping for cardiac support: trends in practice and outcome, 1968 to 1995," Journal of Thoracic and Cardiovascular Surgery, vol. 113, no. 4, pp. 758-769, 1997.

[22] J. Litmathe, P. Feind, U. Boeken, and E. Gams, "Mechanical heart support using intraaortic balloon counterpulsation. A retrospective view and current perspectives," Acta Cardiologica, vol. 59, no. 2, pp. 159-164, 2004.

[23] P. Menon, P. Totaro, A. Youhana, V. Argano, Z. S. Meharwal, and N. Trehan, "Reduced vascular complication after IABP insertion using smaller sized catheter and sheathless technique," European Journal of Cardio-Thoracic Surgery, vol. 22, no. 3, pp. 491-493, 2002.

[24] É. Pinet, E. Cibula, and D. Donlagić, "Ultra-miniature allglass Fabry-Pérot pressure sensor manufactured at the tip of a multimode optical fiber," in Fiber Optic Sensors and Applications V, vol. 6770 of Proceedings of SPIE, pp. 1-8, Boston, Mass, USA, October 2007.

[25] E. Cibula, S. Pevec, B. Lenardili, É. Pinet, and D. Donlagić, "Miniature all-glass robust pressure sensor," Optics Express, vol. 17, no. 7, pp. 5098-5106, 2009.

[26] R. A. Atkins, J. H. Gardner, W. N. Gibler, et al., "Fiber-optic pressure sensors for internal combustion engines," Applied Optics, vol. 33, no. 7, pp. 1315-1320, 1994.

[27] T. Bae, R. A. Atkins, H. F. Taylor, and W. N. Gibler, "Interferometric fiber-optic sensor embedded in a spark plug for in-cylinder pressure measurement in engines," Applied Optics, vol. 42, no. 6, pp. 1003-1007, 2003.

[28] C. Belleville and G. Duplain, "Fabry-Pérot optical sensing device for measuring a physical parameter," US patent no. 5202939, 1993.

[29] C. Belleville and G. Duplain, "Fabry-Pérot optical sensing device for measuring a physical parameter," US patent no. 5392117, 1995.

[30] R. Van Neste, C. Belleville, D. Pronovost, and A. Proulx, "System and method for measuring an optical path difference in a sensing interferometer," US patent no. \#2004/0075841 A1, 2004.
[31] J. F. Mulligan, "Who were Fabry and Pérot?" American Journal of Physics, vol. 66, no. 9, pp. 797-802, 1998.

[32] M. Chavko, W. A. Koller, W. K. Prusaczyk, and R. M. McCarron, "Measurement of blast wave by a miniature fiber optic pressure transducer in the rat brain," Journal of Neuroscience Methods, vol. 159, no. 2, pp. 277-281, 2007. 

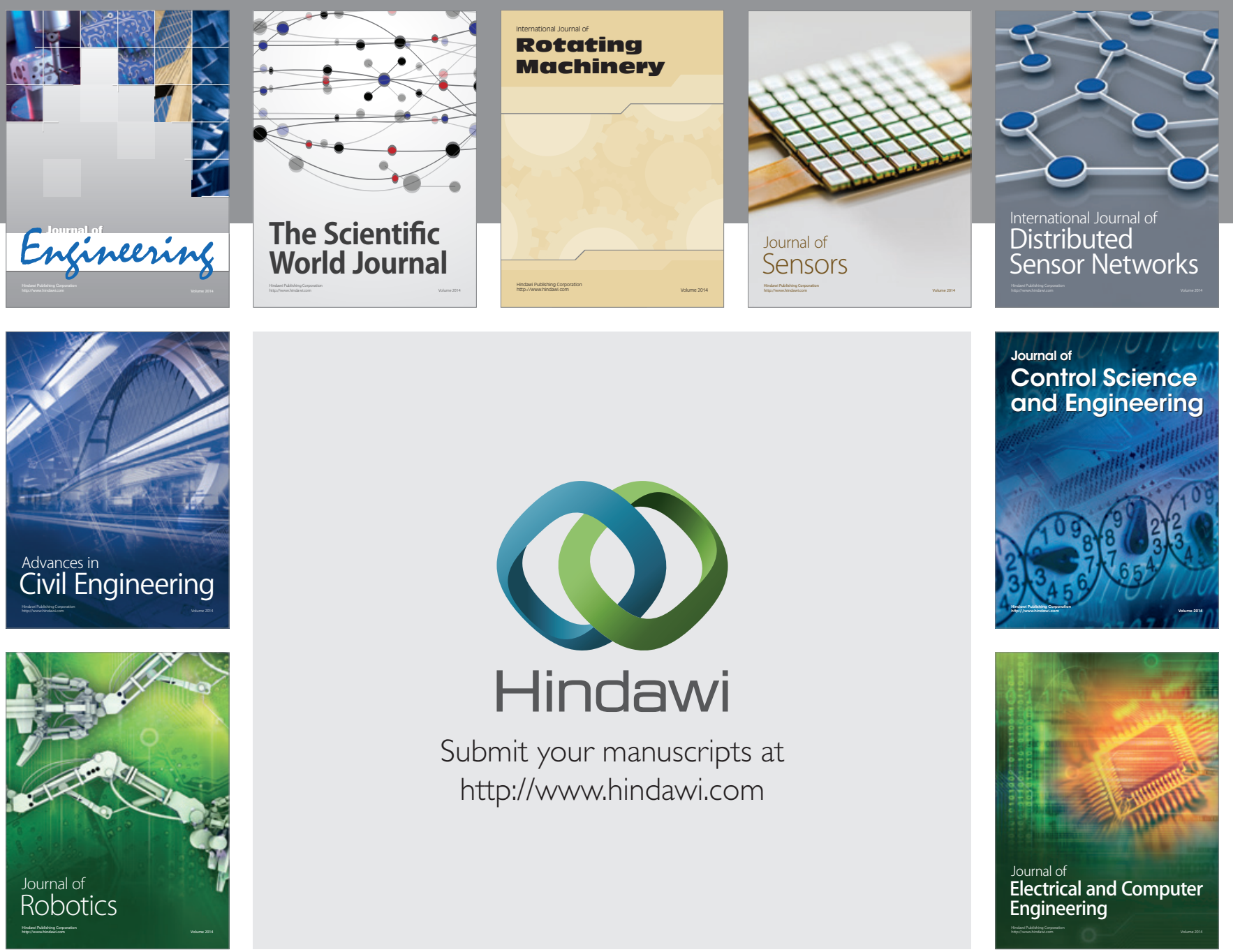

Submit your manuscripts at

http://www.hindawi.com
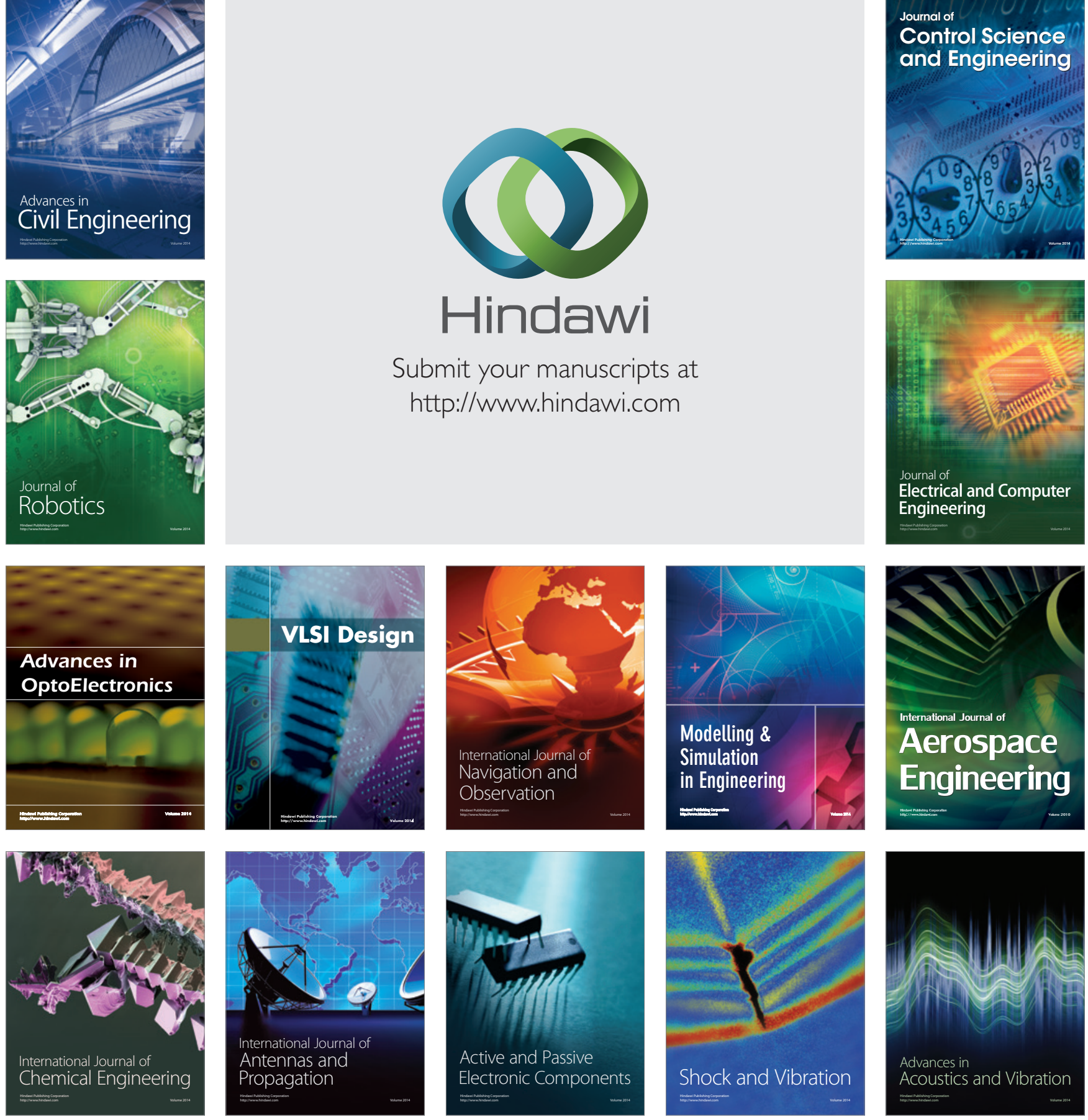